\title{
Prevalence of late gadolinium enhancement in magnetic resonance imaging of patients with left ventricular non-compaction cardiomyopathy
} and Christian Hamm

Address: Kerckhoff-Heart-Center, Bad Nauheim, Germany

* Corresponding author

from 13th Annual SCMR Scientific Sessions

Phoenix, AZ, USA. 21 -24 January 2010

Published: 21 January 2010

Journal of Cardiovascular Magnetic Resonance 2010, I2(SuppI I):PI 95 doi:I0.I I86/I532-429X-I2-SI-PI 95

This abstract is available from: http://jcmr-online.com/content/I2/SI/PI95

(c) 2010 Wilhelm et al; licensee BioMed Central Ltd.

\section{Introduction}

Left ventricular non-compaction cardiomyopathy (LVNC) is a comparatively rare finding which has similar morphological features in cadiac magnetic resonance imaging (cMRI) as those found in other myocardial disorders.

\section{Purpose}

The purpose of our study was to define additional morphological criteria to the already known non-compaction/ compaction ratio described by Jenni et al..

\section{Methods}

14 patients ( 11 male, average age 37 years) with positive Jenni-criterion for LVNC in echocardiography underwent cMRI (Siemens Sonata 1.5 T). Cine TruFISP, TSE T1- (pre and post contrast Early Gadolinium Enhancement ratio (EGEr) according to Friedrich et al. 1998) and T2wheighted images (Edema Ratio (ER - myocardial signal intensity (SI)/scelettal muscle (SI)) were acquired as well as additional late gadolinium enhancement images (LGE) after administration of $0.2 \mathrm{mmol}$ gadodiamide per $\mathrm{kg}$ body weight. LGE was deemed as either beeing present or not.

\section{Results}

Mean EF 45\% ( \pm 18$),$ EDV $174( \pm 88) \mathrm{ml}$ and ESV $107( \pm$ $95) \mathrm{ml}$, Early Gadolinium Enhancement ratio and Edema Ratio had no pathological results in none of the patients $(\mathrm{T} 1=\mathrm{EGEr} \mathrm{3,2} \pm 1.6, \mathrm{~T} 2=\mathrm{ER} 1.6 \pm 0.7) .6$ patients had
LGE and 8 patients had a limited left ventricular ejection fraction. In patients with heart failure 50\% showed LGE, in patients with regular LV-function 30\% showed LGE. LGE had a subendocardial cougar like pattern. 3 patients showed a circuript non-compacted myocardium, 3 patients achieved Jenni-criterion in only one axis-view (long axis or short axis) despite having severly limited ejection fraction, of those 2 had the typical LGE. In 11 patients the whole apex or more than half of left LV-cavity-circumference was concerned. In one case a biventricular affection was found.

\section{Conclusion}

CMR is well applicable for detection of LVNC. LGE might be an additional criterion for diagnosing LVNC in patients with circuript positive Jenni criterion, it seems to define a subgroup with more severely limited LV-EF. 